\title{
How small changes can propel more effective and safe healthcare delivery
}

\author{
Nathan Perlis, MD, MSc, FRCSC \\ Department of Surgery, University Health Network, Princess Margaret Cancer Centre and Toronto General Hospital, University of Toronto, Toronto, ON, Canada
}

Cite as: Can Urol Assoc J 2020;14(8):273. http://dx.doi.org/10.5489/cuai.6820

\section{See related article on page 267}

l n the retrospective study, "Antibiotic prophylaxis for transrectal ultrasound-guided prostate needle biopsy: Compared efficacy of ciprofloxacin vs. the ciprofloxacin/ fosfomycin tromethamine combination,"1 Morin et al demonstrate a steep decline in severe infectious complications when fosfomycin was added to ciprofloxacin for pre-transrectal ultrasound (TRUS) biopsy prophylaxis. Although the rate was as low as $1.1 \%$ with ciprofloxacin alone, I applaud the group for initiating a systemwide change to try and drive the rate even lower. Impressively, by simply adding a single dose of fosfomycin to ciprofloxacin, the urosepsis rate dropped to $0.2 \%$ and no ciprofloxacin-resistant pathogens were identified in the combination group. Although there are several alternatives for reducing TRUS-related infections (e.g., intramuscular aminoglycoside, rectal swab directed therapy, transperineal biopsy), this regimen is particularly attractive, and I believe can be practice-changing for many urology groups. Because ciprofloxacin and fosfomycin were both given as single doses two hours prior to biopsy, it is very convenient for patients and does not require any training or new resources for the physician performing the biopsy.
One main limitation of the current study, which was mentioned in the manuscript, was that patients with urosepsis presenting to a hospital outside the group's practice may not have been captured. However, this would likely be expected to have occurred in similar numbers between groups and may not affect the overall conclusion, although the absolute rates may be slightly higher than presented in the study. Regardless, I think this is an important real-world demonstration of how small changes in clinical practice can propel more effective and safe healthcare delivery.

Competing interests: The author reports no competing personal or financial interests related to this work.

\section{Reference}

1. Morin A, Bergevin M, Rivest N, et al. Antibiotic prophylaxis for transrectal ultrasound-guided prostate needle biopsy: Compared efficacy of ciprofloxacin vs. the ciprofloxacin/fosfomycin tromethamine combination. Can Urol Assoc J 2020;14:267-72. http://dx.doi.org/10.5489/cuai.6248

Correspondence: Dr. Nathan Perlis, Department of Surgery, University Health Network, Princess Margaret Cancer Centre and Toronto General Hospital, University of Toronto, Toronto, ON, Canada; Nathan.Perlis@uhn.ca 